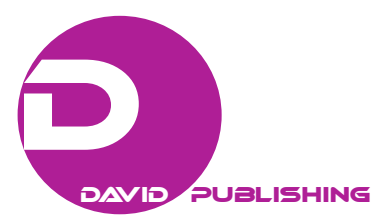

\title{
Strategy for Safer Agricultural Robots
}

\author{
Eleftheria Mitka \\ Kimmeria Campus, Xanthi, Greece
}

\begin{abstract}
Given the prediction that in 2050 agriculture will be doubled due to increasing earth population, the noticeable research question that arises is how do we incorporate safety in agricultural robots? Yet, due to the lack of standardization in this domain, the author will review well-established standards such as autonomous farm equipment, cab-less tractors, and forestry vehicles. Since these robots have come to reduce skilled labour, input costs, and environmental impact, this paper strives to emphasize the need of standardization and regulation framework that will step up the successful commercialization of heave farm machines. The upper goal is to establish a safety assessment improving the compliance with standards and taking account of the public trustworthiness in these emerging applications worldwide.
\end{abstract}

Keywords: precision agriculture, robots, standardization, safety, risk assessment

\section{Introduction}

The farming era represents a crucial part of society due to the attractiveness of the sector and the contribution to human survival. Agricultural field of robotics is both challenging and progressive segment. By 2050 the world's population is likely to increase to 9.6 billion, which will consequently increase by $70 \%$ the demand for fruit and vegetables, according to current estimations (Folley, 2011). Standardization and legal framework will lead to safe machine creation in protected horticulture, forestry, and parkland. Figure 1 explores the existing classes of robotics domain. Notwithstanding that precision agriculture is considered crucial and it is from the most hazardous industries. Agriculture involves highly automated machines that fulfil boring tasks such as de-weeding, seedbed preparation, scouting, fertilization, harvesting with fitted intelligence and associated perception relieving the operator from hard working conditions and musculoskeletal disorders (Figure 2). Harvester reduces spillage and users stress increasing maximum up-time, accuracy and productivity protecting farmer's investment. When it comes to crops, robot has embedded computers with high algorithms on decisional level in order to move around autonomously at the end of row, operating water treatment and waste management and to be monitored by laptop or portable tablet. Self-driving tractors are usually low cost integrated with navigation across crop row in farms while individually operating ploughing and irrigation, haying and chopping fast and robust. Recent technology requires real-time positioning data, on-board laser, and share wireless communication such as cooperation of robots intended for precise pesticide and herbicide (Emmi, Gonzalez-de Soto, Pajares, \& Gonzalez-de Santos, 2014). Otherwise, when cellular communication is unavailable an optical modem can be used for satellite mode capable in centimetre accuracy. 3G Coverage data calculate the complete

Eleftheria Mitka, Ph.D. candidate/Deng, MEng, MBA, Department of Electrical \& Computer Engineering, Kimmeria Campus, Xanthi, Greece.

Correspondence concerning this article should be addressed to Eleftheria Mitka, Department of Electrical \& Computer Engineering, Kimmeria Campus, Building B, Office 2.13, Xanthi GR-67100, Greece. 
field coverage by row-crop planting, harvesting, or seeding and enhance overlap control significantly. That reduces fuel costs and improves precise ground compaction. A display is embedded that alerts the farmer with manage robot health, shows multiple machines simultaneously, tractors real-time logistics information saving time and seed. Furthermore, the display monitors weather data like humidity, wind speed and direction, and temperature in order to advise your crew.

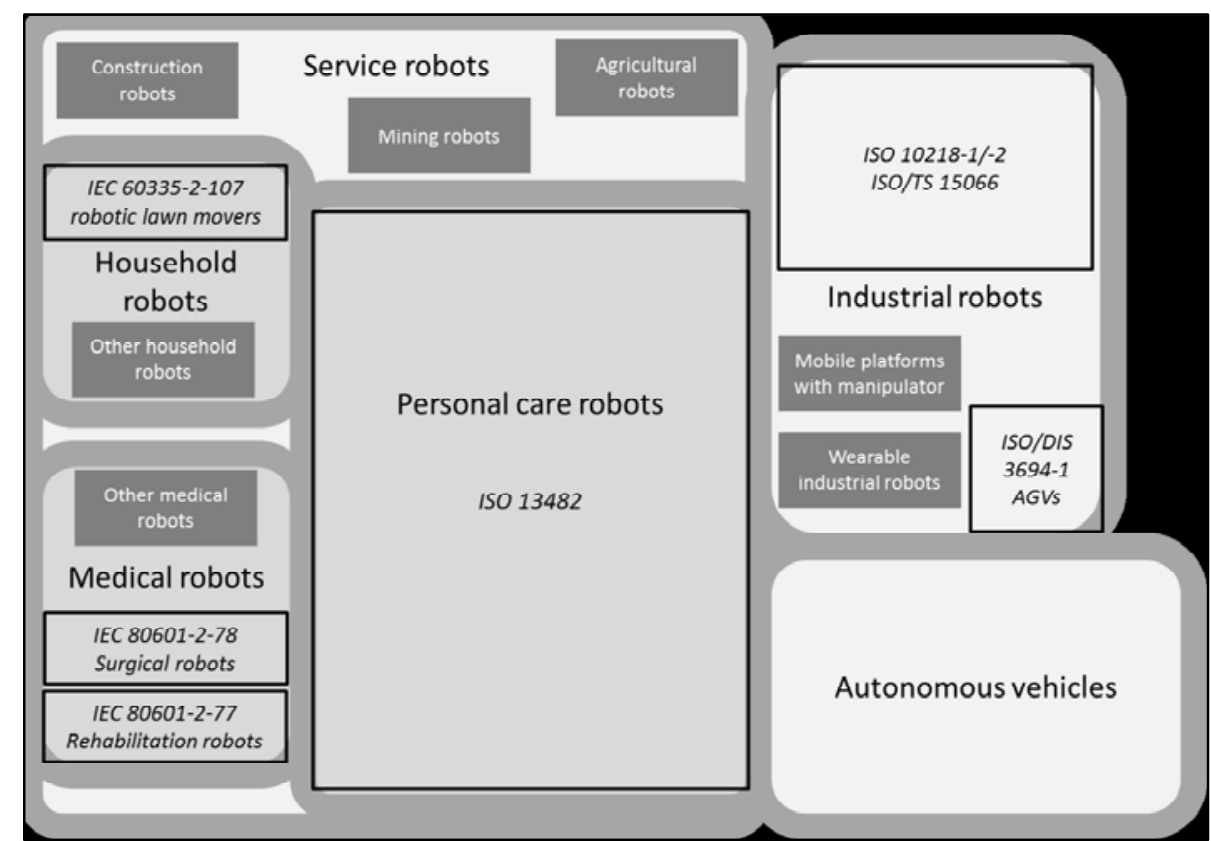

Figure 1. The subclass of agricultural robots as a part of service robotics in the whole community of robots according to ISO/TR 23482-2 draft (ISO/DTR 23482-2, n.d.).

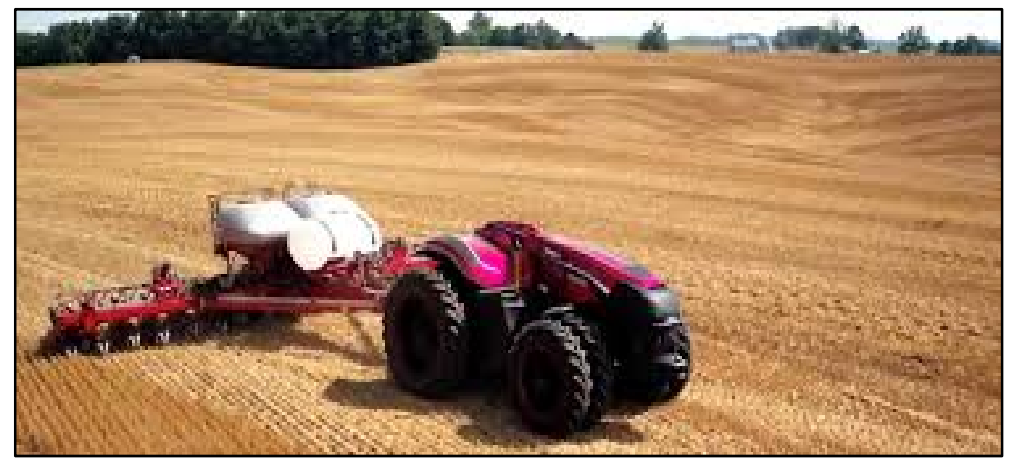

Figure 2. Autonomous vehicle customized for seeding and hydroponic growing system. Source: www.utahbusiness.com.

In the next decades, self-propelled equipment with intelligent farming will cover the rarefaction of skilled labour and add value offering rootworm protection. Most of them incorporate the form of insect and crawl on mechanical legs or mowing wheels driving by two motors or solar panels in order to be eco-friendly. They intended to scan symmetry of leaves size, analyse soil compaction, get ready for cultivation with no or restricted human intervention and auto-steering even in low-visibility conditions. A lot of them are intended for identifying disease, weed, and insects by infrared while ultrasonic sensors arrays keep them on track assisted by telematics, image sensor, and location tracking. 


\section{Current Standards}

The problem of safety in industrial robotics has been a matter of concern for years, in automotive industry, basically (Mitchell, 2010). However, the answer was to separate the operator for the robot cell with "keep out zones" excluding tasks like maintenance, training and troubleshooting that operator could enter the cell (Taubig, Frese, Hertzberg, Luth, Mohr, Vorobev, \& Walter, 2012). In future farming systems, safety standards involve human-robot cooperation and coexistence so it cannot be applied the "principle of separating". Yet, due to the lack of standardization in this domain, the author will survey the well-established standards such as autonomous vehicle ones. Moreover, ISO 13482 (ISO 13482, 2014) can assist the risk assessment, verification and is significant in overall functional safety. Another standard is ISO 25119-2 (ISO 25119-2, 2010) based on quality assurance of ISO 9001 (ISO 9001, 2015) which defines mitigation measures and requirements for operators and designers for tractors and field machines. Protective measures are described in ISO 61496 (ISO 61496, 2012), electro-sensitive protective equipment, defining how fault shall force the system to enter a safe-mode in vision of industrial settings and how multiple faults shall not influence the aforementioned action. Notwithstanding the fundamental ideas of standardization, the procedures for safety of machinery, as well as the ISO 10218-1 (ISO 10218-1, 2011) providing guidelines for robot-human interactions, should also be applied to agronomy for sustainable development.

All these high-level requirements must be achieved in an open-ended chaotic environment, in farms. IEC 61499 can prove very helpful in formal specification deriving implementation (IEC 61499-1, 2012). ISO/DIS 18497 (ISO/DIS 18497, 2014) describes different level of performance for agricultural equipment establishing the increasing level of hazard counting hardware, mean time to repair, fault diagnosis system and traditional faults. The loose connection in standardization towards hardware specifications can be linked with ISO 13849-1 (ISO 13849-1, 2015) and for ethernet communication architecture for tractors the ISO 11783 (ISO 11783, 2017). ISO 26262 is component-level in address human hazards and risks in electronics (ISO 26262, 2011). Finally, the software architecture for safety of agriculture machines can be assessed based on the IEC 61508 (IEC 61508-1, 2010) providing mathematics, diagrams, computed-oriented tools, and formal specification deriving implementation (Ingibergsson, Schultz, \& Kraft, 2015). ISO 14121-1 (ISO 14121-1, 1999) defines the following types of risks: electrical, environmental, hazard by materials and chemical substances, by infrared radiation, by neglecting ergonomic principles in machine, mechanical, vibration-related, noise, radiation.

\section{Risk Assessment}

The flow chart represents the risk assessment for operators, maintenance personnel and apprentices that design and fabric robots safe considering their intended purpose by following this procedure (Figure 3). It also provides a safety strategy based on ISO 13849 (ISO 13849-1, 2015) defining the machine limits for all stakeholders and the type of misuse that leads to dangerous situations. This strategy sums up an iterative process that can be used by standardization's experts and third parties involved, with design measures and, as well as, describing limitations of operators for constant supervision. This strategy involves:

- Risk assessment for each risk according to ISO 14121-1 (ISO 14121-1, 1999) or established standards for industrial robotics, passive safety, safety of electrical equipment, law framework relevant to farms and tractors.

- Determination of machine limits for the intended purpose.

- Identification of the hazards. 
- Risk reduction by inherent measures or by guards.

- Risk estimation.

The quantitative risk estimation is validated counting severity of a harmful incident, possibility to avoid dangerous circumstances and probability of harm occurring. The output variable is risk and arranged within three levels: (1) Low, (2) Medium, and (3) High, as shown in Table 1, counts diverse levels of parameters.

Table 1

The Discrete Levels of Input Variables (ISO 25119, 2010)

\begin{tabular}{|c|c|c|c|}
\hline Severity & Possibility & Probability & Output risk \\
\hline S0-No harm, bruises, scratch & $\begin{array}{l}\text { C0-Controllable/ } \\
\text { operator always correct }\end{array}$ & E0: 0.0001 Improbable & 1. Low \\
\hline S1-Reversible harm & $\begin{array}{l}\text { C1-Easily controllable/ } \\
\text { some operators cannot correct }\end{array}$ & E1: 0.001 Rare events $1 /$ year & 2. Medium \\
\hline S3-Irreversible harm & $\begin{array}{l}\text { C2-Generally controllable/ } \\
\text { average operators correct }\end{array}$ & $\begin{array}{l}\text { E2: } 0.01 \text { Sometimes } \\
<1 \% \text { of operating time }\end{array}$ & 3. High \\
\hline $\begin{array}{l}\text { S4-Fatality/permanent } \\
\text { disability }\end{array}$ & $\begin{array}{l}\mathrm{C} 3-\text { Fatal injuries/operator } \\
\text { never able to correct }\end{array}$ & $\begin{array}{l}\text { E3: } 0.1 \text { Often } \\
<10 \% \text { of operating time } \\
\text { E4: } 1 \text { Frequently } \\
10-100 \% \text { of operating time }\end{array}$ & \\
\hline
\end{tabular}

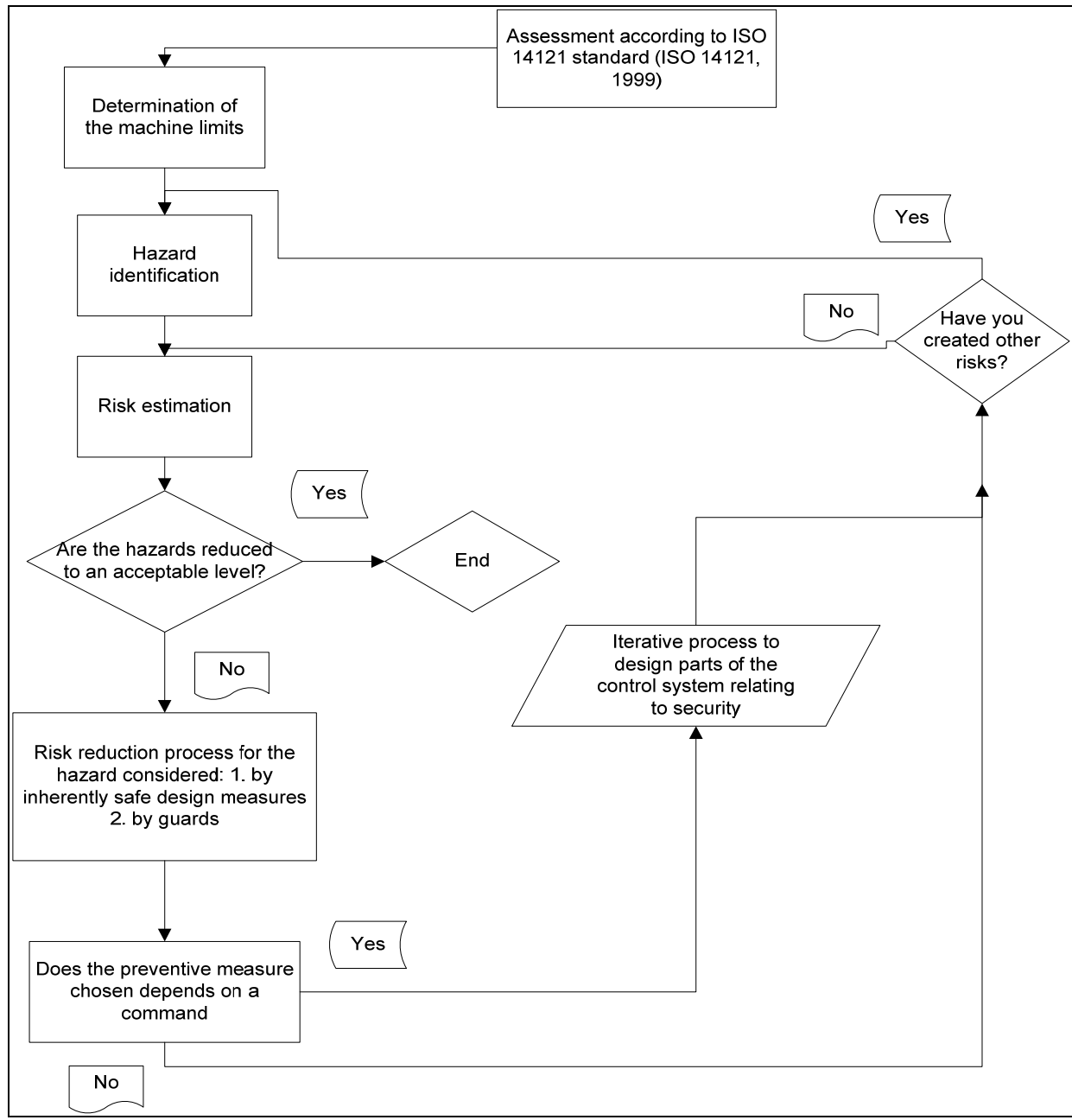

Figure 3. Strategy for certifying safety and support the standardization's framework based on ISO 13849-1 (ISO 13849-1, 2015). 


\section{Safety Requirements}

Taking account of the status-quo of standardization activities towards precision agriculture and the computation of risk, safety restrictions are concentrated so as to apply the outcomes of risk management and ensure safety and reliability of operator and agribot (agricultural robot). The safety precautions for people and all stakeholders are given below for agricultural robots:

Hazard 1: Unexpected start-up

- The manufacturer should take the necessary precautions alerting the operator that regular tests on all protective structures need to be completed. Detailed directions on how to operate these tests should be provided. Unauthorized change of adapters, over speed the engine or rotating driveline is prohibited. The maintenance should be carried out by service experts.

- Resetting only a single button shall be unfeasible. A more complex reset procedure shall be applied to prevent from harmful situations. The target of this requirement is that a simple restart procedure shall not be feasible to solve any arbitrary condition preventing an operator from pushing by mistake a restart button and, as a result, an inappropriate or disoriented start-up most probable leading to an accident.

Hazard 2: Unexpected deceleration/stop

- The restart position shall be tested for its appropriate performance, proper calibration and maintenance.

- An emergency stop shall be embedded and tested that remains functional preventing any hazardous condition (Jiang \& Cheng, 1990).

- Make sure stops will not result in a crushing and the embedded pressure safety button will be start if the robot exceeds its limits because of wrong operator command, malfunction in mechanics or bug in software.

- Detailed directions to the farmer shall be given in order to be able to constantly gather data and guidelines on line.

Hazard 3: Unexpected acceleration

- The tractor shall be prevented from running high speed (Bonney \& Yong, 1985). High speed can result in loss of control, no ability of braking quickly, damage to equipment and components. Transfer the tractor with good visibility in order to be seen by other drivers, use extremity lights or flashers, pull over for traffic and frequently check for approaching from behind.

- Designer should check tractor's procedures naming manual stop, control of user presence and station, manual controller and drive speed.

- Post-manufacture check of the full scale system, about its maximum, minimum, optimal speed and settings, start/end points, path exceptional accuracy, time, save fuel or efficacy criteria, dead distance.

Hazard 4: Unexpected change of direction

- The emergency stop button shall stop within seconds every harmful movement quickly. The emergency stop should be easily accessible and maybe red color should help (IEC 60204-1, 2005).

- It should be stated clearly in the user's guide that operator will ask for help in case any abnormal or disoriented movement observed. Any possible interference shall not lead to loss of calibration or changing direction with no progress.

- The safety buttons, controllers, and sensors should pass dynamic tests and validated that they are in a good condition. 
- The manufacturer should inform the operator that he shall maintain properly in place all guards, shields, buttons, safety features, and sensors. The operator should not turn on the machine if any parts are faulty or do not work.

Hazard 5: Wrong direction at start-up

- The stop and reset switches and process shall be frequently reminded. An audible alert if a shut-off switch stops working and a visual cue or emergency verbal exclamation, should be embedded if needed. The operator needs to be informed about secondary guidelines to halt and how to return to start position.

- The software shall embed a self-awareness algorithm that detects any arbitrary function in the field. If that program does not bear, the operator should not control a shut-off of a suspicious robot when possible.

- Field machine shall incorporate an emergency button to halt the motion on the remote control so as to block its path if it is arbitrary.

Hazard 6: Failure to start

- Control parameter settings should be aligned towards the specifications while the machine stays immobile or out of service and safety precautions ought to be given to the operator (Jiang \& Cheng, 1990).

- The user's guide shall inform farmer, that he ought to inspect vehicle for weeds or corns, to clean base with a cloth, to ensure contact among station cables and power supply unit, to make sure that power supply is plugged at main power and check that coiled cord is placed in its place properly, to check the good reliability of the power cable to the connector and to confirm reliable connection in cables.

Hazard 7: Failure to stop

- Children and untrained people must not ride upon, misuse or attack the equipment. Operator shall pay attention to infants since they are careless and in a weak position. Pets shall be prevented from tragic accidents when hunting the robot or disturbing its task.

- Operator ought to possess a checklist of guidelines regarding the equipment's operation in a noticeable position in the farm.

- Ensure that all ranchers are well out of robot's motion path when starting the machine.

- Control a "return-to-home" function following deliberate control-link transmission failure.

- Warning signs of electrical shock shall be placed if needed to warn careless or unauthorized humans that may be careless.

Hazard 8: Failure to react on the command

- Some field robots are able to execute its task with restricted or no supervision. In some cases, limited control may be applied though remote control.

- According to the capabilities of the robot, it generally ought to be as solid as feasible with smallest chassis and heaviness, so as to maneuver effectively in the farm.

- The robot shall be equipped with an audio or visual cue in order to be recognizable in case of changing its path or task.

Hazard 9: Avoid damaging people, infrastructure, and crops

- The machine should be appropriately placed and arm the tools so that operator could halt the motion if a tool is mishandled. Embed a sensor to alert the operator of the mistreated tool and provide an indicator naming a strong noise or visible alert, to warn farmers and ranchers that a tool is misused.

- Do not stay in touch with the machine as it operates. Forbid farmers from expelling fruits or plants on the machine or tools while it executes among the crop rows. 
- Communication between robots is necessary when multiple robots are operating on a farm. Multiple machines result in three levels of interaction: motion coordination, frequent communication, and collaboration. It shall be feasible to embed diverse communication protocols, plug and play features, software and hardware minimization that result to modularity and expandability. Vehicles with sprayer, autonomous harvester, truck and heterogeneous tractors can participate in the small team of multi-tractor system.

- All cables and wires must be embedded inside the chassis of the robot to avoid electrical shock.

Hazard 10: Robustness against noise and uncertainties

- The field robot should not be affected by interferences such as high ambient noise, in field radio signal, unshielded computers, infrared remote controllers and magnetic fields in order to ensure reliability and robustness in harsh conditions.

- The operator should ensure that any sensory equipment accompanied with the device is located not close to extreme light, microwave antennas, magnetic waves, vibration, heat, and sound (Jenkins, 1993).

- The robot shall be equipped with a specific audio or visual signal with a frequency that is not within the range of noise frequencies, in case of an audio signal.

Hazard 11: Recognize worn out tools

- Every faulty condition inside the machine should be recognized while the field equipment should carry on working if it does not violate the safety precautions.

- Except for spare of not well-preserved or auxiliary objects, the robot should not demand routine or unexpected treatment more than a time in a year.

Hazard 12: Quick detection and isolation of faults

- The programming architecture that enforces safety constraints on agribot should take into account that programs must not be affected by faults in operation system.

- A specialized diagnostics program ought to be installed so that it will figure out the root cause of a fault condition. The absence of a complete process might result in arbitrary handling rather than really solve the mismanagement.

- Use of well-established programming languages, where possible with a certified compiler. Avoiding error prone code features such as dynamic obstacles, corn, automatic type etc. Use of style guidelines and structured programming methodologies. Architectures where process software and integrity checks run independent from each other.

Hazard 13: Low modelling and low computational requirements

- The software shall divide the program in minor operational loops, the loops shall be divided with sequences and iterations, a restricted number of paths ought to be added in programming, complicated ramifications and leaps shall be prevented, modules shall be connected to input specification settings, and complex calculations should be avoided when making decisions on forks and loops.

- Machine task path simulation in 2-dimension or 3-dimension should be executed from manufacturer to imitate robot motion in an urgent situation. Since divergences among actual and the cybernetic world might take place, simulation information ought to be checked on the real prototypes.

- The manufacturer should check 3D georeferenced position and velocity control, person and object detection, path planning, obstacle avoidance, selective muting of safeguards, application of varying protection fields based on situation, safety-related autonomous decision. The field boundary information is well-known, as well as if it is 
planted or no, the wet and dry yield, total yield, average moisture, seeding variety, and the machine location where the tractor can travel with coordinated motion.

- The computers of the main controller shall be connected to the network with point-to-point links considering the amount of sensors, peripherals, operating systems and computing power.

Hazard 14: Manual resetting

- Operator shall be informed about how to select manual resetting, verify that switching the external.

- Control does not lead to dangerous conditions, and how to prevent the event of loss of external control.

- Supportive tutorials and help guides should require practical guidelines, so that operator can have immediate communication in data on how to restart the machine and with a comprehensive knowledge how the agribot can operate its path.

- The values and parameters for the recovery procedure shall be tested, as it is crucial to reset the robot after a malfunction. A standby operation system ought to be triggered by programming in an emergency (Jiang \& Cheng, 1990).

- If remote controller is shut off, it should continuous with manual mode (Izard, Ribeiro, Barreiro, \& Valero, 2011).

- Expanding path offset and orientation offset, it should be continuous with manual mode.

Hazard 15: A lock to prevent switching on the system by accident

- Hardware control system and/or safety program should be initiated when pressing the machine to halt in an urgent condition, to protect from dangerous events or tearing of tools.

- Embedded diagnostic software should not lead to fail shutting off, in an emergency situation.

- Commands with redundant power for agribot stored in Random Access Memory should be tested so that a power outage would not result in a corruption of program.

- Agribot's parts shall use only backup in Read Only Memory to avoid altering the operational program.

Hazard 16: Leakage of moisture of liquids

- If overheated gasses, liquids, or combustible substances such as pesticide are contained in tanks inside the robot, the designer shall ensure that any increase of temperature will not cause fire. In case of fire, shut-off the equipment instantly, run away smoke, and if it is extended do not try to extinguish it.

- Chemical burn can be harmful in case that battery is mistreated and explosion hazard or leaking of corrosive liquid, if the battery is incorrectly placed. In particular, goggles, gloves, and respirator must be used for protection.

- Moisture content of the soil is another external condition influencing the performance of the robots. Moisture content can be measured by static sensors placed on strategic positions in the field identified by Autotrack guidance. The broad-acre spray for herbicide shall stop under strong wind conditions. The broadcast spray of herbicide should avoid chemical applications on cotton plants or sides without unwanted weeds. The robot distinguishes slight differences among colour and leaves as the machine passes learning as it goes.

- A visual daily check shall be implemented in case of leakage and that every safety features are in place. Hazard 17: Software or camera failure

- Software crashes: Computer halts via hardware watchdog.

- Problems with camera including: no image, frozen image, wrong field of view, artefacts on display, image 
which does not display, scenery (vary light), and delay image (Izard, Ribeiro, Barreiro, \& Valero, 2011).

- Software shall embed all safety guidelines preventing from all hazards from bruises to blindness.

\section{Conclusion}

In conclusion, researchers rely only on machine learning and artificial intelligence and forget machine ethics, risk assessment and hierarchy among safety goals which is a paradox. The author proposes a strategy of safety assessment with a set of safety rules based on scientific knowledge and well-established standards as hoping to fill the standardization gap of these robots. Safety requirements shall be understandable and easily readable by humans reflecting the trade-off among safety goals. The agricultural robot should be able to react itself on unforeseen events like low sun, rain and snow, sloping terrain or hilly ground combining encoders, gyros, sonar and cameras. Verification and validation using computer vision and neural networks is still an open issue. Not-too-distant future work concerns regulation framework of multi-faceted robotics community using Artificial Neural Networks that they are going to play a significant role beyond "Asimovian" ethics (Asimov, 1950).

\section{References}

Asimov, I. (1950). I, robot. NY: Gnome Press.

Bonney, M. C., \& Yong, D. (1985). Robot safety. UK: International Manufacturing Technology. IFS Publications Ltd.

Emmi, L., Gonzalez-de Soto, M., Pajares, G., \& Gonzalez-de Santos, P. (2014). New trends in robotics for agriculture: Integration and assessment of a real fleet of robots. The Scientific World Journal, 2014, 1-21.

Foley, J. A. (2011). Can we feed the world and sustain the planet? Scientific American, 305, 60-65.

IEC 60204-1. (2005). 10.7.1 Devices for emergency stop shall be readily accessible.

IEC 61508-1. (2010). Functional safety of electrical/electronic/programmable electronic safety-related systems-Part 1: General requirements.

IEC 61499-1. (2012). Function blocks-Part 1: Architecture.

ISO/DTR 23482-2. (n.d.). Robotics-Application of ISO 13482-Part 2: Application guide.

ISO 14121-1. (1999). Safety of machinery. Risk assessment. Part 1: Principles.

ISO 10218-1. (2011). Robots for industrial environments-safety requirements-Part I: Robot.

ISO 9001. (2015). Quality management systems-Requirements.

ISO 13849-1. (2015). Safety of machinery. Safety-related parts of control systems. Part 1: General principles for design.

ISO 11783. (2017). Tractors and machinery for agriculture and forestry-Serial control and communications data network. Part 1: General specifications.

Ingibergsson, M. T. J., Schultz, P. U., \& Kraft, D. (2015). Towards declarative safety rules for perception specification architectures. International Workshop on Domain-Specific Languages and Models for Robotic Systems, September 28, Hamburg (Germany).

Izard, G. M., Ribeiro, A., Barreiro P., \& Valero, C. (2011). Safety functional requirements for robot fleets for highly effective agriculture and forestry management. Proceedings of the First International Workshop on Robotics and Associated High Technologies and Equipment for Agriculture. France.

Jiang, B., \& Cheng, O. (1990). A procedure analysis for robot system safety. International Journal of Industrial Ergonomics, 6(2), 95-117. Industrial Engineering Department, Auburn University, AL 36849, USA.

Jenkins, F. (1993). Practical requirements for a domestic vacuum-cleaning robot. Technical Report.

Mitchell, L. R. (2010). Toyota's lesson: Software can be unsafe at any speed. Computerworld.

Taubig, H., Frese, U., Hertzberg, C., Luth, C., Mohr, S., Vorobev, E., \& Walter, D. (2012). Guaranteeing functional safety: Design for provability and computer-aided verification. Autonomous Robots, 32(3), 303-331.

TC 22. (2011). Road vehicles functional safety. International Organization for Standardization. International Standard ISO $26262,2011$.

TC 23. (2010). Tractors and machinery for agriculture and forestry-Safety-related parts of control systems. International Standard ISO 25119, 2010. 
TC 23. (2014). Agricultural machinery and tractors-Safety of highly automated machinery. International Standard ISO/DIS 18497, ISO, 2014.

TC 44. (2012). Safety of machinery-electro-sensitive protective equipment. International Electronical Commission. International Standard IEC 61496, 2012.

TC 184. (2014). Robots and robotic devices-Safety requirements for personal care robots. International Organization for Standardization. International Standard ISO 13482, 2014. 